Louisiana State University

LSU Digital Commons

Faculty Publications

Department of Physics \& Astronomy

$1-1-1993$

\title{
Unconventional charge gap formation in FeSi
}

\author{
Z. Schlesinger \\ IBM Thomas J. Watson Research Center \\ Z. Fisk \\ University of California, San Diego \\ Hai Tao Zhang \\ University of California, San Diego \\ M. B. Maple \\ University of California, San Diego \\ J. DiTusa \\ Nokia Bell Labs
}

See next page for additional authors

Follow this and additional works at: https://digitalcommons.Isu.edu/physics_astronomy_pubs

\section{Recommended Citation}

Schlesinger, Z., Fisk, Z., Zhang, H., Maple, M., DiTusa, J., \& Aeppli, G. (1993). Unconventional charge gap formation in FeSi. Physical Review Letters, 71 (11), 1748-1751. https://doi.org/10.1103/

PhysRevLett.71.1748

This Article is brought to you for free and open access by the Department of Physics \& Astronomy at LSU Digital Commons. It has been accepted for inclusion in Faculty Publications by an authorized administrator of LSU Digital Commons. For more information, please contact ir@lsu.edu. 


\section{Authors}

Z. Schlesinger, Z. Fisk, Hai Tao Zhang, M. B. Maple, J. DiTusa, and G. Aeppli 


\section{UC Irvine}

\section{UC Irvine Previously Published Works}

\section{Title}

Unconventional charge gap formation in FeSi.

Permalink

https://escholarship.org/uc/item/8515c4ht

\section{Journal}

Physical review letters, 71(11)

ISSN

0031-9007

\section{Authors}

Schlesinger, $Z$

Fisk, Z

Zhang, HT

et al.

\section{Publication Date}

1993-09-01

\section{DOI}

10.1103/physrevlett.71.1748

License

https://creativecommons.org/licenses/by/4.0/4.0

Peer reviewed 


\title{
Unconventional Charge Gap Formation in FeSi
}

\author{
Z. Schlesinger \\ IBM T.J. Watson Research Center, Yorktown Heights, New York 10598
}

\author{
Z. Fisk, Hai-Tao Zhang, and M. B. Maple \\ University of California, San Diego, La Jolla, California 90000
}

\section{J. F. DiTusa and G. Aeppli}

AT\&T Bell Labs, Murray Hill, New Jersey 07974

(Received 3 March 1993)

\begin{abstract}
We report the first infrared and optical measurements of the absolute conductivity of FeSi. Above $200 \mathrm{~K}$ the ac conductivity of FeSi resembles that of a dirty metal, while at low temperature most of the conductivity below $60 \mathrm{meV}$ is depleted, consistent with an energy gap of that magnitude. Thus in contrast to what is expected from a picture based on simple thermal activation, the gap disappears at a temperature which is low relative to its size. Moreover, the spectral weight lost below the gap does not reappear just above the gap, but is instead distributed over an energy range of order $\mathrm{eV}$.
\end{abstract}

PACS numbers: $71.28 .+\mathrm{d}$

FeSi is a nonmagnetic narrow gap semiconductor [1] with unusual features that it shares with a class of rare earth compounds known as hybridization gap semiconductors or Kondo insulators [2]. At low temperature, the electrical resistivity $\rho(T)$ and magnetic susceptibility $\chi(T)$ of FeSi follow thermal activation laws characteristic of barriers of order $0.1 \mathrm{eV}$. At higher temperature ( $T \gtrsim 300 \mathrm{~K}) \rho(T)$ rises slowly with $T$, as in a metal, and $\chi(T)$ passes through a maximum near $500 \mathrm{~K}$ [1]. If a Curie-Weiss description is used for $\chi(T)$ above its maximum, then the effective moment per $\mathrm{Fe}$ is several Bohr magnetons. While local density functional band structure calculations [3] yield a semiconducting gap of roughly the correct size $(\sim 0.1 \mathrm{eV})$, they cannot account for the large magnitude of $\chi(T)[4,5]$, nor the result that the substitution of as little as $10 \%$ of the $\mathrm{Fe}$ by Co yields a magnet with helical spin order [6]. Thus with regard to spin fluctuations there are substantial discrepancies with conventional band theory. A question which naturally arises is whether an examination of the charge fluctuations would yield similar discrepancies. We have thus performed infrared and optical reflectivity measurements to establish the temperature dependent evolution of the charge fluctuation spectrum of FeSi. While a number of rare earth and actinide-based Kondo metals and semiconductors have been characterized in this way [7-10], these are the first such measurements on FeSi. Two aspects of our data are particularly unusual and interesting. The temperature dependence shows a disappearance of the gap at lower temperature than would be expected based on a simple thermal activation picture. Even more striking, the spectral weight lost on gap formation at low temperature is spread over an energy range of order $\mathrm{eV}$ ( $\sim 30$ times the gap). In this sense, band theory fails spectacularly, even though it yields roughly the correct value for the infrared gap.

The samples investigated were single crystals of typical size $2 \times 2 \times 2 \mathrm{~mm}^{3}$, grown by vapor transport. Figure 1 shows the magnetic susceptibility $\chi(T)$ and resistivity $\rho(T)$ measured for crystals of the same type used in the infrared study. The solid lines represent the activated forms, $\chi(T)=(C / T) e^{-\Delta_{x} / K T}$ and $\rho(T)=\rho_{0} e^{-\Delta_{g} / 2 K T}$, with $\Delta_{\chi}=650 \mathrm{~K}$ and $\Delta_{g}=695 \mathrm{~K} ; \rho_{0}=0.04 \mathrm{~m} \Omega \mathrm{cm}$ and $C \simeq 1.8$
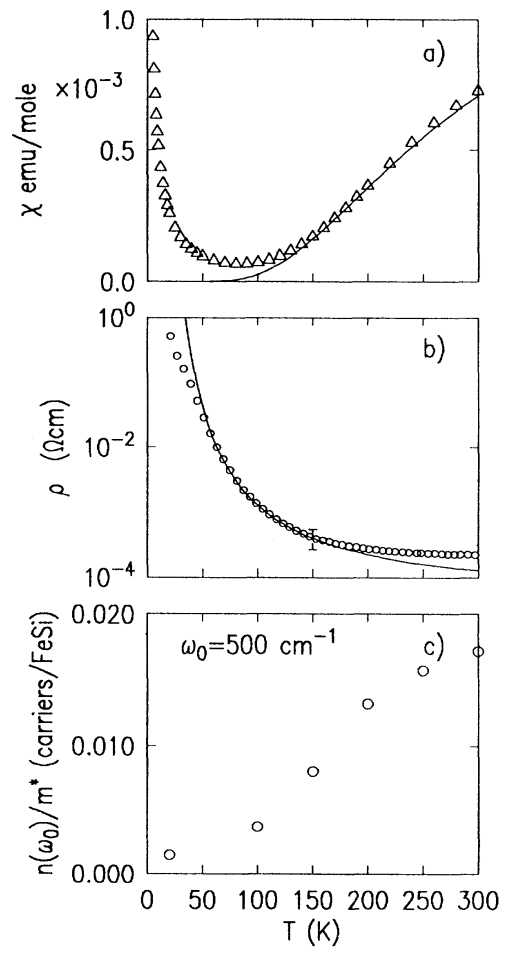

FIG. 1. Magnetic susceptibility $\chi$, the resistivity $\rho$, and the integral of the infrared conductivity from 0 to $500 \mathrm{~cm}^{-1}, \mathrm{n} / \mathrm{m}^{*}$, are shown as a function of temperature for cubic $\mathrm{FeSi}$. The solid curves in parts (a) and (b) are activated forms, as described in the text. 
emu $\mathrm{K} / \mathrm{mole}$. The Curie constant $C$ is that associated with $g \simeq 2$ and $s=\frac{3}{2}$, which might occur for a $d^{7}$ ion such as $\mathrm{Fe}^{+}[11]$. The high quality of our crystals is demonstrated by the large resistance ratio, $\rho(1.2 \mathrm{~K}) /$ $\rho(300 \mathrm{~K}) \sim 10^{5}$, and the small size of the low temperature Curie tail in $\chi(T)$, which corresponds to $0.2 \%$ of spin $\frac{3}{2}$ impurity per formula unit.

Near normal incidence reflectivity measurements of the cubic FeSi crystals were performed using a scanning interferometer for the $50-5000 \mathrm{~cm}^{-1}$ range, and a grating spectrometer for the near infrared and visible range (2000-25000 $\mathrm{cm}^{-1}$ ), as described previously [12]. The samples were abrasively polished using water as the lubricant, and mounted in a circulating He cryostat, in which all the reflectivity measurements were performed without breaking the sample vacuum. An evaporated Ag film immediately adjacent to the sample was used as a reference. The sample and reference were mounted on a stable moving arm, with which their positions could be reproducibly interchanged within each temperature and frequency range. The absolute accuracy with this technique is about $1 \%$; however, the precision for measuring temperature dependent changes in $R$ is better than $0.3 \%$. Repeated cycling of the temperature from 20 to $250 \mathrm{~K}$ was employed to probe for temperature dependence up to 20000 $\mathrm{cm}^{-1}$.
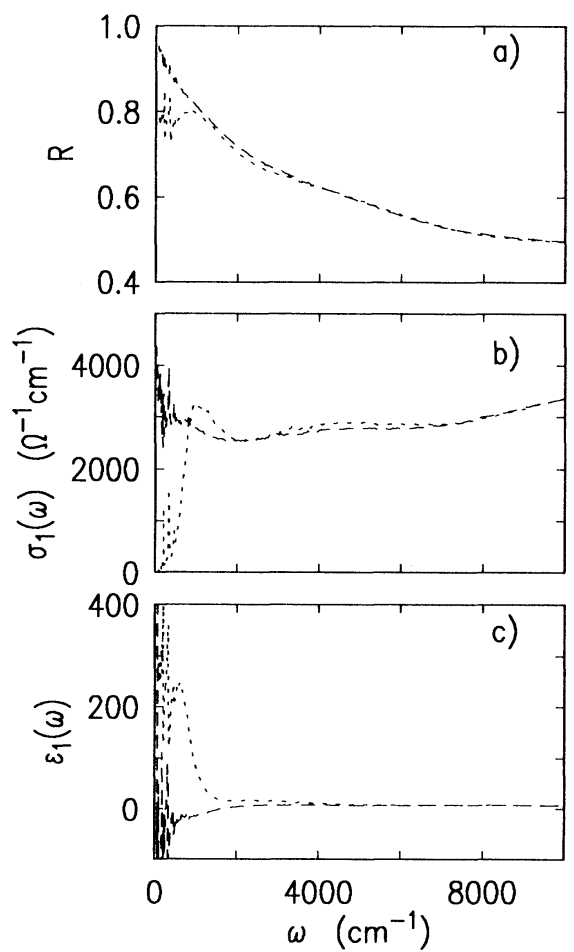

FIG. 2. Measured reflectivity, the real part of the conductivity $\sigma_{1}(\omega)$, and dielectric function $\epsilon_{1}(\omega)$ of $\mathrm{FeSi}$ are shown as a function of frequency at 250 (long dashes) and $20 \mathrm{~K}$ (short dashes) $\left(10000 \mathrm{~cm}^{-1} \simeq 1.25 \mathrm{eV}\right)$.
In Fig. 2, the infrared reflectivity, conductivity $\left[\sigma_{1}(\omega)\right]$, and dielectric function $\left[\epsilon_{1}(\omega)\right]$ of an FeSi crystal are shown for temperatures of 20 and $250 \mathrm{~K}$ up to $8000 \mathrm{~cm}^{-1}$. Figure 3 shows the temperature dependence of the reflectivity and of $\sigma_{1}(\omega)$ at low frequency, as well as the indefinite integral of $\sigma_{1}(\omega), n(\omega) / m^{*}$. Note the excellent correspondence between the low frequency infrared conductivity and the dc values [from Fig. 1(b)] shown by the symbols along the $\omega=0$ axis in Fig. 3(b).

The conductivity data are obtained by Kramers-Kronig transform [13] using reflectivity data from 80 to 22000 $\mathrm{cm}^{-1}$. At low frequency, terminations with $R=1$ $-a \omega^{1 / 2}$ or $R=$ const are matched to the data. These terminations have a negligible effect on the data above $\sim 120 \mathrm{~cm}^{-1}$. The method of high frequency termination is more significant, and as such we have explored a wide
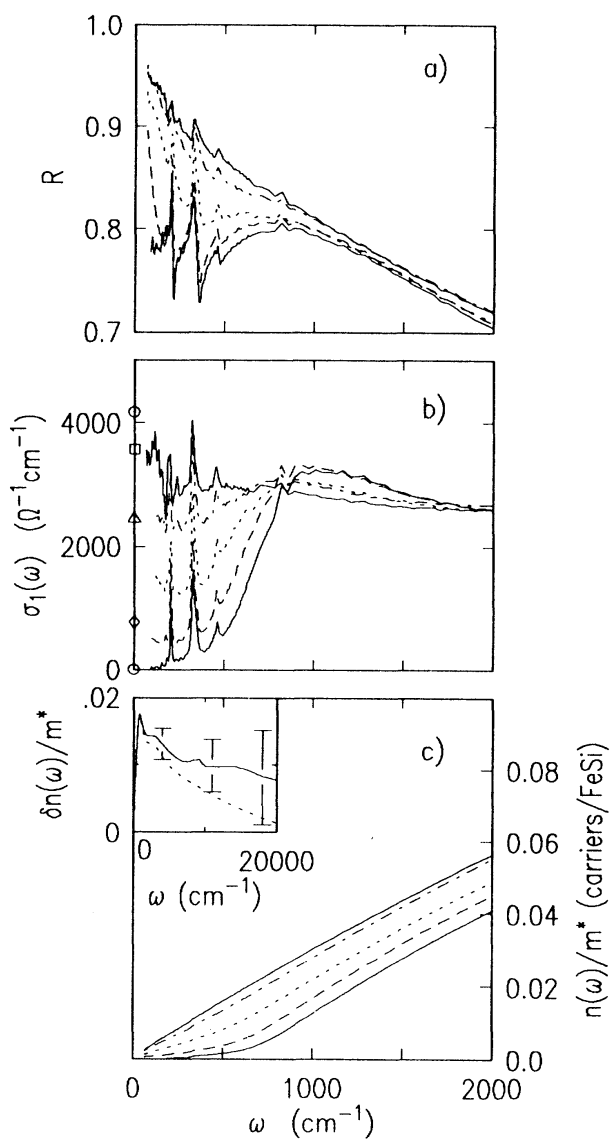

FIG. 3. Infrared reflectivity $R$, conductivity $\sigma_{1}(\omega)$, and the integral of the conductivity from 0 to $\omega, n(\omega) / m^{*}$, are shown as a function of frequency for $T=20$ (solid), 100 (dashed), 150 (dotted), 200 (dot-dashed), and $250 \mathrm{~K}$ (solid) for cubic $\mathrm{FeSi}$. The symbols at $\omega=0$ show the measured values of $\sigma_{\mathrm{dc}}$ [from Fig. 1(b)] for the same temperature sequence. The inset shows the difference between $n(\omega) / m^{*}$ at 250 and at $20 \mathrm{~K}$ (solid curve). The dotted curve shows the effect on $n(\omega) / m^{*}$ shifting the $20 \mathrm{~K}$ reflectivity data by $+0.5 \%$. 
variety of possible terminations and analyzed their effects on the data. To avoid pathological cases, we restrict our attention to extensions of the data which correspond to a conductivity in the ultraviolet comparable (within a factor of 2 ) to that of elemental transition metals such as $\mathrm{Fe}$ or $\mathrm{Ni}[14,15]$. This is appropriate since the UV transitions involve core levels, and are little influenced by environment or banding effects [13]. We find that the absolute value of the conductivity shows some dependence on the termination (primarily above $10000 \mathrm{~cm}^{-1}$ ). However, the juxtaposition of the conductivity spectra at different temperatures, which embodies our central results, is unaffected by the choice of termination.

At $250 \mathrm{~K}$, the absence of a sharp Drude peak at the origin distinguishes FeSi from elemental transition metals, where $\sigma_{\mathrm{dc}}$ is typically $\sim 100000 \Omega^{-1} \mathrm{~cm}^{-1}$ at room temperature. The conductivity thus resembles that of a dirty metal; however, to account for the low conductivity by ordinary disorder (elastic scattering) would require an elastic mean free path of about $0.5 \mathrm{~nm}$ (assuming one carrier per $\mathrm{Fe}$ ). This is unrealistic given the high quality of our crystals $(\sim 1$ defect per $500 \mathrm{FeSi}$ pairs $)$. The data instead suggest that strong inelastic scattering is limiting the conductivity, as it does at high temperatures in rareearth based heavy fermion metals [9] due to the strong carrier-moment interaction.

At low frequency the characteristics of gap development are observable in both the reflectivity and $\sigma_{1}(\omega)$ at low temperature (Fig. 3). One also notices that the phonon related features in the reflectivity become increasingly prominent as the metallic screening disappears at low temperature. At $20 \mathrm{~K}$ the conductivity is very small below $500 \mathrm{~cm}^{-1}$, and there is a dramatic onset of absorption between 500 and $700 \mathrm{~cm}^{-1}$. The dielectric function $\epsilon_{1}(\omega)$ rises to a very large value $(-300)$ at low frequency and temperature, which is primarily the residue of the $\sim 500 \mathrm{~cm}^{-1}(60 \mathrm{meV})$ gap feature in $\sigma_{1}(\omega)$. The peaks in $\sigma_{1}(\omega)$ at 205,332 , and 466 and possibly $820 \mathrm{~cm}^{-1}$, which are particularly easy to see at low temperature due to the lower noise level in $\sigma_{1}(\omega)$, are due to direct absorption by infrared active optical phonons. The strength of the $332 \mathrm{~cm}^{-1}$ mode is unusually large, as discussed below.

From these data we estimate a characteristic energy scale for charge excitations of $500 \mathrm{~cm}^{-1}(60 \mathrm{meV})$, which is the frequency at which the steeply increasing part of $\sigma_{1}(\omega)$ extrapolates to zero [Fig. 3(b)]. Thus we ignore the tail on $\sigma_{1}(\omega)$ extending to lower energy, which may be associated with impurities and/or absorption due to an imperfect surface. The activated behavior of $\rho_{\mathrm{dc}}$ suggests that it is appropriate to associate the $500 \mathrm{~cm}^{-1}$ threshold $(\sim 700 \mathrm{~K})$ with a gap in the charge fluctuation spectrum at low $T$. The filling and disappearance of the gap occur at a lower temperature than would be expected from simple thermal activation, since the gap is about $700 \mathrm{~K}$, and has mostly filled in by $200 \mathrm{~K}$. Such a high ra- tio of the characteristic energy to temperature $(\sim 3.5)$ is suggestive of collective behavior. The gap disappears entirely near $300 \mathrm{~K}$, which is roughly the temperature where the slope of $\rho$ vs $T$ becomes positive. One thus observes in both the resistivity and the infrared conductivity a departure from activated behavior at a surprisingly low temperature. This is the first experiment which explores the disappearance of a gap as a function of temperature in a semiconductor, thus a comparison to actual data from a conventional semiconductor is not possible.

One can, however, compare our results for $\mathrm{FeSi}$ to what would be expected for conventional semiconductors. There, thermally excited carriers provide free-carrier conductivity in the gap at high temperature, and also block interband transitions into the states above the gap (which are now partially occupied) thereby reducing the conductivity above the gap [13]. The intensity associated with transitions from states just below the gap, which are partially unoccupied, is similarly reduced. With increasing temperature, a decrease in the conductivity above the gap compensates for the increase in the free-carrier conductivity. An infrared sum rule, which requires that the integrated area under $\sigma_{1}(\omega)$ must be unchanged, is thus satisfied [13]. The expectation for a conventional semiconductor is that this shifting of spectral weight primarily occurs with a frequency range of order several times the gap. Thus the integral of $\sigma_{1}(\omega)$ from $\omega=0$ to a frequency of order several times the gap should be nearly independent of temperature.

For FeSi it is apparent in Figs. 2(b) and 3(b) that the modest conductivity increase just above the gap does not compensate for the conductivity missing from the gap region at low temperature. That $\epsilon_{1}(\omega)$ is large and positive [Fig. 2(c)] establishes that the "missing area" is not shifted to low frequency, as occurs for a superconductor. (The reflectivity in our measurement range would be noticeably influenced if more than $\sim 5 \%$ of the area were shifted to low frequency.) In Fig. 3(c) the integrals of $\sigma_{1}(\omega)$ are nearly parallel between about 700 and 2000 $\mathrm{cm}^{-1}$, indicating very little recovery of missing area in this frequency range. The inset in Fig. 3(c) shows the difference between $n(\omega) / m^{*}$ at 250 and at $20 \mathrm{~K}$. This quantity diminishes gradually with increasing frequency, indicating a slow recovery of the missing area at sufficiently high energy. The growing error bars reflect the numerical uncertainty associated with the fact that $\delta n(\omega)$ is obtained from the integral of the difference between two large numbers. The dotted curve shows the effect of shifting the $20 \mathrm{~K}$ reflectivity upward by $0.5 \%$ with respect to the reflectivity at $250 \mathrm{~K}$. Because of these systemic uncertainties, our data are consistent with a recovery of the missing area by a frequency of $\gtrsim 16000$ $\mathrm{cm}^{-1}(2 \mathrm{eV})$.

Both the broad form of the high temperature conductivity and the observation that one needs to go to unusually high frequency to satisfy the conductivity sum rule 
suggest that the physics of FeSi involves an energy scale much larger than the gap energy. This higher energy scale could be associated with an electron-electron repulsion energy or a conduction bandwidth, which are typically of order several volts for $3 d$ bands. In heavy fermion compounds, the strong scattering (near unitarity limit) of carriers by local moments produces a similarly broad conductivity spectrum $[9,10]$ at high temperature (above $\left.T_{\text {Kondo }}\right)$, and a coherent metallic state forms at low temperature. A well-known feature of the Kondo problem, which is at the root of the heavy fermion phenomenon, is that the ground state wave function describing the screening of the local (Kondo) moment includes contributions from the entire conduction band. Thus the observation that the disappearance of the magnetic moments in $\mathrm{FeSi}$ coincides with extensive rearrangement of the charge fluctuation spectrum is consistent with a Kondo lattice picture for FeSi.

Based on the similarities between the temperature dependence of the magnetic susceptibility and integrated conductivity, shown in Figs. 1 (a) and $1(\mathrm{c})$, it is reasonable to associate the missing spectral weight due to charge gap formation with the magnetic moment lost on cooling. The result that the moment is derived from order 1 electron suggests that we associate the missing area with approximately one carrier. The missing area of $\sim 0.02$ carriers/FeSi would then imply a carrier effective mass of order 50, which is substantially larger than the $m^{*} \simeq 5-10$ from band calculations [3].

Another interesting aspect of the infrared data is the strength of the infrared active phonons. Particularly for the phonon at $332 \mathrm{~cm}^{-1}$, the integrated area under the peak in $\sigma_{1}(\omega)$ is quite large. Strong phonons have also been observed in heavy-fermion-valence fluctuation compounds such as $\mathrm{URu}_{2} \mathrm{Si}_{2}$ and $\mathrm{CePd}_{3}[7,9,10,16]$, where optic phonons at 105 and $118 \mathrm{~cm}^{-1}$ exhibit strengths of approximately 1000 and $600 \mathrm{~cm}^{-1}$, respectively. For comparison, the most intense well-known infrared active phonon occurs in the classic ionic compound $\mathrm{MgO}$ at a frequency of $400 \mathrm{~cm}^{-1}$ with a strength of $\simeq 1000 \mathrm{~cm}^{-1}$ [17]. Phonons of comparable strength have also been documented in cuprates [18]. In the present work, the phonon at $332 \mathrm{~cm}^{-1}$ has a strength of $1300 \mathrm{~cm}^{-1}$, which suggests a highly ionic character for $\mathrm{FeSi}$, as well as phenomenological similarity to $\mathrm{CePd}_{3}$ and $\mathrm{URu}_{2} \mathrm{Si}_{2}$. Such a localized (ionic) character is also indicated by the Curielike high temperature susceptibility, an aspect FeSi also shares with $\mathrm{CePd}_{3}$ and $\mathrm{URu}_{2} \mathrm{Si}_{2}$.

In summary, we have studied the infrared reflectivity and conductivity of $\mathrm{FeSi}$, which exhibit characteristics of a poor metal above $\sim 200 \mathrm{~K}$, and of a small gap semiconductor for $T \lesssim 100 \mathrm{~K}$. The gap disappears at unusually low temperature relative to its size; moreover, the conductivity that is displaced from the gap region does not appear just above the gap, but is instead spread over more than $1 \mathrm{eV}$. Our experiments demonstrate that an ordinary semiconductor picture can no more account for the charge response than for the spin response of FeSi. Taken together, and noting similarities to heavy fermion systems, the anomalous charge and spin behavior suggest that a Kondo insulator description is more appropriate for FeSi.

The authors acknowledge valuable conversations with L. D. Rotter, C. Pickover, B. Bucher, P. Coleman, D. R. Hamann, L. F. Mattheis, D. H. Lee, P. A. Littlewood, M. P. A. Fisher, and especially G. A. Thomas. Research at UCSD was supported by the National Science Foundation under Grant No. DMR91-07698.

[1] V. Jaccarino, G. K. Wertheim, J. H. Wernick, L. R. Walker, and Sigurds Arajs, Phys. Rev. 160, 476 (1967).

[2] G. Aeppli and Z. Fisk, Comments Condens. Matter Phys. 16, 155 (1992).

[3] L. F. Mattheiss and D. R. Hamann (to be published).

[4] D. R. Hamann (private communication).

[5] K. Tajima, Y. Endoh, J. E. Fischer, and G. Shirane, Phys. Rev. B 38, 6954 (1988).

[6] J. Beille, J. Voiron, and M. Roth, Solid State Commun. 47, 399 (1983).

[7] F. E. Pinkerton, A. J. Sievers, J. W. Wilkins, M. B. Maple, and B. C. Sales, Phys. Rev. Lett. 47, 1018 (1981).

[8] G. Travaglini and P. Wachter, Phys. Rev. B 29, 893 (1984).

[9] B. C. Webb, A. J. Sievers, and T. Mihalisin, Phys. Rev. Lett. 57, 1951 (1986).

[10] D. A. Bonn, J. D. Garrett, and T. Timusk, Phys. Rev. Lett. 61, 1305 (1988).

[11] A. Abragam and Brebis Bleaney, Electron Paramagnetic Resonance of Transition Ions (Oxford, London, 1970).

[12] Z. Schlesinger, R. T. Collins, F. Holtzberg, C. Feild, G. Koren, and A. Gupta, Phys. Rev. B 41, 11237 (1990).

[13] Frederick Wooten, Optical Properties of Solids (Academic, San Francisco, 1972).

[14] H. Ehrenreich, H. R. Phillip, and D. J. Olechna, Phys. Rev. 131, 2469 (1963).

[15] A. P. Lenham and D. M. Treherne, in Optical Properties and Electronic Structure of Metals and Alloys, edited by F. Abeles (North-Holland, Amsterdam, 1966).

[16] L. J. Sham and J. W. Wilkins, Phys. Rev. B 30, 3062 (1984).

[17] D. H. Martin, Adv. Phys. 14, 39 (1965); We define strength as $f_{i}$, in the notation of this reference.

[18] R. T. Collins, Z. Schlesinger, G. V. Chandrashekhar, and M. W. Shafer, Phys. Rev. B 39, 2251 (1989). 